\title{
A Simple Bumpless Transfer Mechanism with Application to Multi-Effect Evaporators
}

\author{
B.J. Burke, A.J. Rojas, J.S. Welsh and G.J. Adams
}

\begin{abstract}
In many applications one needs to switch between control laws. This requires some form of bumpless transfer. Here we propose a simple bumpless mechanism for possible implementation on a set of Multi-Effect Evaporators in a Sugar Mill located in Queensland, Australia. The ultimate goal of this study is to allow on-the-fly testing of different controller designs and exchanging of control authority to achieve increased performance.
\end{abstract}

\section{INTRODUCTION}

Bumpless transfer (BT) is generally a requirement when switching between controllers or control authority in an industrial process. Without a BT mechanism a difference between the process input and the controller output can arise when one controller is replaced by another. Controller substitution can be motivated, for example, during plant startup procedures when, for instance, to reach the operating point a controller with good tracking properties is required and once at the operation point a controller with good regulation properties is desired. Here the startup controller can be switched to an alternative controller used for normal operation. Controller substitution can also be used when controlling an inherently nonlinear process around a finite number of selected operation points using gain scheduling. Finally, control law substitution can result from the need for on-line testing of different control designs.

In process control, a closely related problem involves the use of integral action. A possible drawback can be that, due to changes in operation or sudden disturbances, the controlled process actuator saturates, and the true process input $u_{T}(t)$ is (for a time) different from the controller output $u(t)$. As a result, the controller error signal can wind up resulting in the difference between the process input and the controller output increasing. This can lead to severe control difficulties. To avoid such problems, most control solutions include some form of anti-wind-up (AW) mechanism. Essentially these mechanisms disconnect the integral part of the controller when the input is subject to saturation.

Schemes that try to avoid, or minimize, the difference $\Delta_{u}(t)=u_{T}(t)-u(t)$ are generally called BT schemes. Early results can be found in [7], where the authors introduce a conditioning technique (see also [10], [11]). The authors of [8] introduce a gain scheduled controller design method

ARC Centre of Excellence for Complex Dynamic Systems and Control, School of Electrical Engineering and Computer Science, The University of Newcastle, Australia. Emails: Brendan.Burke@studentmail. newcastle.edu.au, Alejandro.Rojas@newcastle.edu. au, James.Welsh@newcastle. edu.au, Gregory. Adams@ newcastle.edu.au which implements a BT mechanism. More recently [4] derives a BT mechanism motivated by an hybrid dynamical system viewpoint. The problem of avoiding, or minimizing, $\Delta_{u}(t)$ has also been studied under the framework of linear matrix inequalities (LMI) [9], $L_{2}$ optimization [13], [14], Linear Quadratic (LQ) theory [12] and $H_{\infty}$ control [3], [15].

Our motivation to study BT schemes comes from the requirement of testing different control designs on the last stage controllers for level and sugar concentration (brix) of the multi-effect evaporators (MEE) stage at Pioneer Sugar Mill, a CSR owned sugar mill near Townsville, North Queensland, Australia. We also want to achieve BT when swapping control authority from the inflow to the outflow for the level controller, and from the outflow to the inflow for the brix controller. A possible reason for swapping control authority, when in nominal operation, is that it can provide (for example) tighter brix control. Although industrial applications of successful BT schemes have been reported in the literature, see for example [2], complexity of the solution in [2] is a concern and simplicity is highly desired.

Our first contribution is then the development of a very simple BT mechanism by adapting an existing anti-windup (AW) scheme used at Pioneer Mill. The proposed antiwind-up bumpless transfer (AWBT) scheme ensures that the dynamics of the latent controller are driven by the true process input $u_{T}$. The proposed AWBT scheme can be seen to be closely related to the ideas presented in [5]) and to be a particular case of the AWBT scheme proposed in [6].

Our second contribution is then to modify the AWBT scheme to allow BT for the case of control authority swapping. The control authority swapping AWBT (SW-AWBT) scheme is, to the best knowledge of the authors, a novel contribution.

We verify the effectiveness of the proposed AWBT and SW-AWBT solutions through simulations of the MEE stage. The schemes proposed here require minimal controller modification to guarantee the safety and application by the plant operators, in line with the arguments presented in [6].

The paper is organised as follow: In Section II we present a short review of the MEE model. In Section III we describe the proposed modifications to implement the BT methods such to ensure $\Delta_{u}(t) \approx 0$. In Section IV we show the application and efficacy of the proposed methods for the MEE model using simulations. Finally, in Section V, we give final remarks and discuss future research directions. 


\section{Multi EFFECt Evaporators}

A model of the multi-effect evaporators at Pioneer mill was developed in [1]. This model development is summarised below.

A total energy balance for the system is given by

$\frac{d}{d t}\left(M_{\text {total }} H_{\text {total }}\right)=\left(h_{s}-h_{c}\right) S+h_{\text {in }} F_{\text {in }}-h_{v} V-h F_{\text {out }}$,

where ${ }^{1} M_{\text {total }}$ is the mass of both solute and steam, $H_{\text {total }}$ is the total enthalpy content in solute and steam, $S$ is the inflow of steam, $h_{s}$ is the enthalpy of the steam, $h_{i n}$ is the enthalpy of the inflowing juice, $V$ is the vapour flow, $h_{v}$ is the enthalpy of the vapour, $h$ is the enthalpy of the outflowing juice and $h_{c}$ is the energy content in the condensated steam. We consider a static balance, that is $d\left(M_{\text {total }} H_{\text {total }}\right) / d t=0$. This is justified given the relatively slow dynamics related to the heating of liquid. This leads to the following simplified energy balance:

$$
0=\left(h_{s}-h_{c}\right) S+h_{\text {in }} F_{\text {in }}-h_{v} V-h F_{\text {out }} .
$$

Rearranging (2),

$$
\begin{aligned}
V & =\frac{1}{h_{v}}\left\{h_{\text {in }} F_{\text {in }}-h F_{\text {out }}+\left(h_{s}-h_{c}\right) S\right\} \\
& =\frac{h_{\text {in }}}{h_{v}} F_{\text {in }}-\frac{h}{h_{v}} F_{\text {out }}+\frac{\left(h_{s}-h_{c}\right)}{h_{v}} S .
\end{aligned}
$$

We see that $V$ is linearly dependent on the inflow rate $F_{\text {in }}(t)$, the outflow rate $F_{\text {out }}(t)$ and the steam flow $S(t)$.

These considerations, and a simple mass balance on juice, lead to the following model ${ }^{2}$ linking inflow $F_{\text {in }}(t)$, outflow $F_{\text {out }}(t)$ and juice level $L(t)$ (as a percentage of total evaporator chamber height).

$$
\frac{d L}{d t}=\frac{1}{E_{\text {vol }}}\left\{F_{\text {in }}(t)-F_{\text {out }}(t)-O(t)\right\}
$$

where $E_{v o l}$ denotes the evaporator volume and

$$
O(t)=V(t) f(L(t))
$$

is the actual vapour flow. Substituting for the vapour flow (3) in (4) gives

$$
\begin{aligned}
& \frac{d L}{d t}=\frac{1}{E_{\text {vol }}}\left\{\left(1-f(L(t)) \frac{h_{\text {in }}}{h_{v}}\right) F_{\text {in }}(t)-\right. \\
& \left.\left(1-f(L(t)) \frac{h}{h_{v}}\right) F_{\text {out }}(t)-f(L(t)) \frac{\left(h_{s}-h_{c}\right)}{h_{v}} S(t)\right\} .
\end{aligned}
$$

The second variable of interest is brix $(B(t))$, the sugar concentration inside the evaporator (which, assuming perfect mixing, is the same as brix in the outflow). To develop an

\footnotetext{
${ }^{1}$ The mass and flow quantities in (1) are dependent on time, however the time dependence is not shown in (1) for clarity; enthalpies are assumed constant.

${ }^{2}$ Time-dependent variables will now have the $(t)$ qualifier, including $V$; enthalpies are still constant.
}

expression for $B(t)$, we first obtain an expression for sugar volume, $C(t)$, in the evaporator:

$$
\frac{d C}{d t}=B_{\text {in }}(t) F_{\text {in }}(t)-B(t) F_{\text {out }}(t),
$$

where $B_{i n}(t)$ is the brix level in the inflow. Now the brix level is related to the sugar volume according to $C(t)=$ $L(t) E_{\text {vol }} B(t)$. Hence

$$
\begin{aligned}
\frac{d C}{d t}=\frac{d}{d t}\left(L(t) E_{\text {vol }} B(t)\right) & =E_{\text {vol }}\left(L(t) \frac{d B}{d t}+B(t) \frac{d L}{d t}\right) \\
& =B_{\text {in }}(t) F_{\text {in }}(t)-B(t) F_{\text {out }}(t) .
\end{aligned}
$$

Rearranging (7), and substituting (4), gives

$$
\frac{d B}{d t}=\frac{1}{L(t) E_{v o l}}\left\{F_{\text {in }}(t)\left(B_{\text {in }}(t)-B(t)\right)+B(t) O(t)\right\} .
$$

Substituting for $O(t)$ from (5) gives

$$
\begin{aligned}
\frac{d B}{d t}= & \frac{1}{L(t) E_{v o l}}\left\{\left(B_{\text {in }}(t)-B(t)\left(1-f(L(t)) \frac{h_{i n}}{h_{v}}\right)\right) F_{\text {in }}(t)\right. \\
& \left.-B(t) f(L(t)) \frac{h}{h_{v}} F_{\text {out }}(t)+B(t) f(L(t)) \frac{\left(h_{s}-h_{c}\right)}{h_{v}} S(t)\right\} .
\end{aligned}
$$

This equation shows that rate of change of brix is not affected by outflow, except through the vapour flow term.

The MEE at Pioneer Mill consists of two sets of six effects each. In this paper we only consider the last stage evaporator, whilst lumping together the five previous evaporators in one fictitious effect. Thus the model, using (6) and (8), is

$$
\begin{aligned}
\frac{d L_{1}}{d t}= & \frac{1}{E_{v o l 1}}\left\{\left(1-f_{1}\left(L_{1}(t)\right) \frac{h_{i n 1}}{h_{v 1}}\right) F_{e s j}(t)-\right. \\
& \left.\left(1-f_{1}\left(L_{1}(t)\right) \frac{h_{1}}{h_{v 1}}\right) F_{o u t 1}(t)-f\left(L_{1}(t)\right) \frac{\left(h_{s 1}-h_{c 1}\right)}{h_{v 1}} S_{i}(t)\right\} \\
\frac{d B_{1}}{d t}= & \frac{1}{L_{1}(t) E_{v o l 1}}\left\{\left(B_{e s j}(t)-B_{1}(t)\left(1-f_{1}\left(L_{1}(t)\right) \frac{h_{i n 1}}{h_{v 1}}\right)\right) F_{e s j}(t)\right. \\
- & \left.B_{1}(t) f_{1}\left(L_{1}(t)\right) \frac{h_{1}}{h_{v 1}} F_{\text {out } 1}(t)+B_{1}(t) f_{1}\left(L_{1}(t)\right) \frac{\left(h_{s 1}-h_{c 1}\right)}{h_{v 1}} S_{i}(t)\right\} \\
\frac{d L_{2}}{d t}= & \frac{1}{E_{v o l 2}}\left\{\left(1-f_{2}\left(L_{2}(t)\right) \frac{h_{i n 2}}{h_{v 2}}\right) F_{i n 2}(t)-\right. \\
& \left.\left(1-f_{2}\left(L_{2}(t)\right) \frac{h_{2}}{h_{v 2}}\right) F_{\text {out } 2}(t)-f_{2}\left(L_{2}(t)\right) \frac{\left(h_{s 2}-h_{c 2}\right)}{h_{v 2}} O_{1}(t)\right\} \\
\frac{d B_{2}}{d t}= & \frac{1}{L_{2}(t) E_{v o l 2}}\left\{\left(B_{i n 2}(t)-B_{2}(t)\left(1-f_{2}\left(L_{2}(t)\right) \frac{h_{i n 2}}{h_{v 2}}\right)\right) F_{i n 2}(t)\right. \\
- & \left.B_{2}(t) f_{2}\left(L_{2}(t)\right) \frac{h_{2}}{h_{v 2}} F_{\text {out } 2}(t)+B_{2}(t) f_{2}\left(L_{2}(t)\right) \frac{\left(h_{s 2}-h_{c 2}\right)}{h_{v 2}} O_{1}(t)\right\} \\
O_{1}(t)= & \left(\frac{h_{i n 1} h_{v 1}}{h_{v s j}} F_{e s j}(t)-\frac{h_{1}}{h_{v 1}} F_{\text {out } 1}(t)+\frac{\left(h_{s 1}-h_{c 1}\right)}{h_{v 1}} S_{i}(t)\right) f_{1}\left(L_{1}(t)\right) \\
O_{2}(t)= & \left(\frac{h_{i n 2}}{h_{v 2}} F_{i n 2}(t)-\frac{h_{2}}{h_{v 2}} F_{o u t 2}(t)+\frac{\left(h_{s 2}-h_{c 2}\right)}{h_{v 2}} O_{1}(t)\right) f_{2}\left(L_{2}(t)\right),
\end{aligned}
$$

where the coupling takes place by means of $O_{1}$ and $F_{e s j}$ is the flow of juice from the upstream effect supply tank. Note that $F_{\text {out } 1}=F_{i n 2}$ and that $B_{1}=B_{i n 2}$. The subindex 1 is used for the lumped stage model, whilst the subindex 2 is used for the final stage evaporator; see Figure 1. 


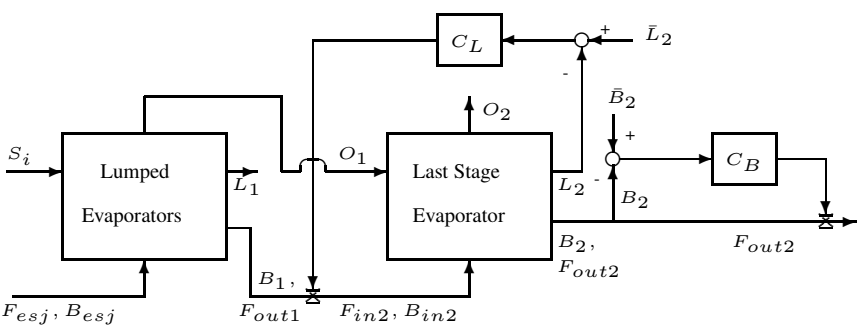

Fig. 1. Control architecture at Pioneer Mill; $\bar{L}_{2}$ and $\bar{B}_{2}$ are level and brix setpoints for the final evaporator.

\section{Typical Control Architecture}

A typical architecture to regulate evaporators at Pioneer Mill is shown in Figure 1. Optimal heating efficiency is attained at a certain juice level inside each evaporator, requiring good level control. At Pioneer Mill, controllers manipulate both the inflow and outflow to the final evaporator unit in each set, as well as the flows between each unit.

Pioneer Mill is a co-generation plant, which supplies power to the national electricity grid under contract. The ability to deliver the contracted power can be compromised by unsatisfactory operation of the evaporators, due to the important role the evaporation process plays in sugar milling.

One aspect of the operation of the evaporators at Pioneer Mill is the presence of high frequency oscillations in brix occurring after crushing mill stoppages. The cause of these oscillations has been found to be due to non-linear flow characteristics at low flows causing a form of stiction. This can be avoided somewhat by reversing the brix and level controls so that the level (a less critical control variable) is controlled via the outflow valve, and the brix is controlled via inflow adjustments.

Indeed, this configuration is used at other CSR sugar mills, and in some cases the evaporators start up in one configuration, and are required to be swapped to the second configuration during the mill startup procedure.

Thus for Pioneer Mill we desire a mechanism for switching bumplessly between controller configurations. The bumpless transfer between controllers will also be invaluable for testing of new controllers.

\section{Bumpless TRANSFer Mechanism}

In this section we take a simple AW scheme and adapt it for use in a BT configuration. Let the control law have transfer function $C(s)$. The controller $C(s)$ is assumed minimum phase, to be bi-proper and contain an integrator.

Since $C(s)$ is stably invertible we have that $C^{-1}(s)$ is a stable biproper transfer function. We can thus write

$$
C^{-1}(s)=g_{o}+H(s),
$$

where $H(s)$ is stable and strictly proper. We then implement the control law as shown in Figure 2, where we also include plant input saturation limits. Notice, from Figure 2, that in a linear regime (i.e. no saturation) we have

$$
\frac{U(s)}{E(s)}=\frac{g_{o}^{-1}}{1+g_{o}^{-1} H(s)}=\frac{1}{g_{o}+H(s)}=C(s),
$$

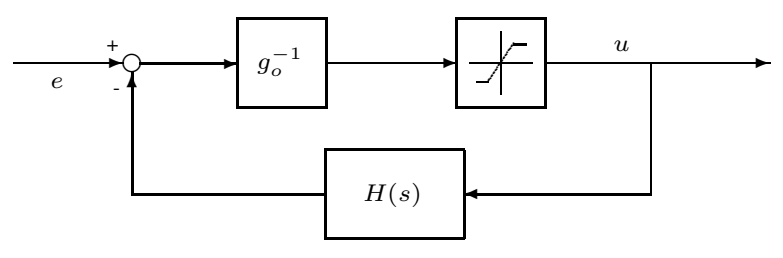

Fig. 2. Anti-wind-up mechanism at Pioneer Mill.

as required. Next we change the architecture of Figure 2 to achieve BT by using the arrangement in Figure 3. We see that the states of $C(s)$ are driven by $u_{T}$ the true process input.

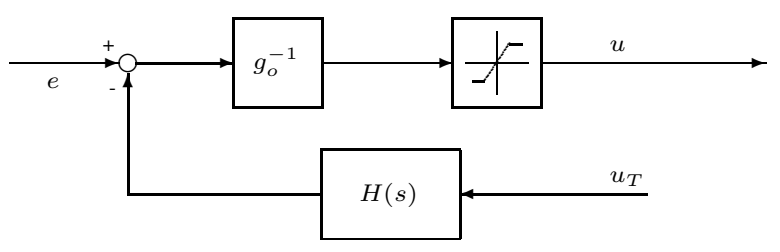

Fig. 3. Anti-wind-up scheme as a BT mechanism.

In Figure 4, we then present the implementation of the proposed AWBT scheme for the level control loop. The proposed scheme switches between two level controllers $C_{L a}(s)$ and $C_{L b}(s)$. When the switch is in position $a$ we have that $C_{L a}(s)$ is the active controller and $C_{L b}(s)$ is the latent controller. Vice versa, when the switch is in the $b$ position then $C_{L b}(s)$ is the active controller and $C_{L a}(s)$ is the latent controller. In Figure 4 the switch happens between two different designs for the level controller, that is $C_{L a}$ and $C_{L b}$. An entirely equivalent scheme (not shown) can be implemented if the user requires to test two different designs for the brix controller, that is $C_{B a}$ and $C_{B b}$. The achievement of a bumpless transfer when switching between different controller design can be clearly observed in the first simulation presented in the next section.

We now move to the second objective that motivated as to study the implementation of an AWBT scheme in the context of MEE control, namely the concept of control authority swapping. Control authority swapping for a process such as an MEE can be motivated by the fact that during start-up we can achieve a faster response of the process variables if the inflow is used for level control and outflow is used for brix control. On the other hand, when in nominal operation around the setpoints $\bar{L}_{2}$ and $\bar{B}_{2}$, the effective control of brix becomes paramount. A more reactive and responsive brix control can then be achieved if the inflow is used for brix control and the outflow is used for level control, reversing the initial control authorities.

In Figure 5, we present an extension of the AWBT scheme that implements a control authority swapping. The control authority swapping AWBT (SW-AWBT) scheme switches the control authority for the inflow $F_{\text {in }}$ between the level controller $C_{L}(s)$ and the brix controller $C_{B}(s)$. It also (although not shown in Figure 5) simultaneously switches the control authority for the outflow $F_{\text {out }}$ between the brix 


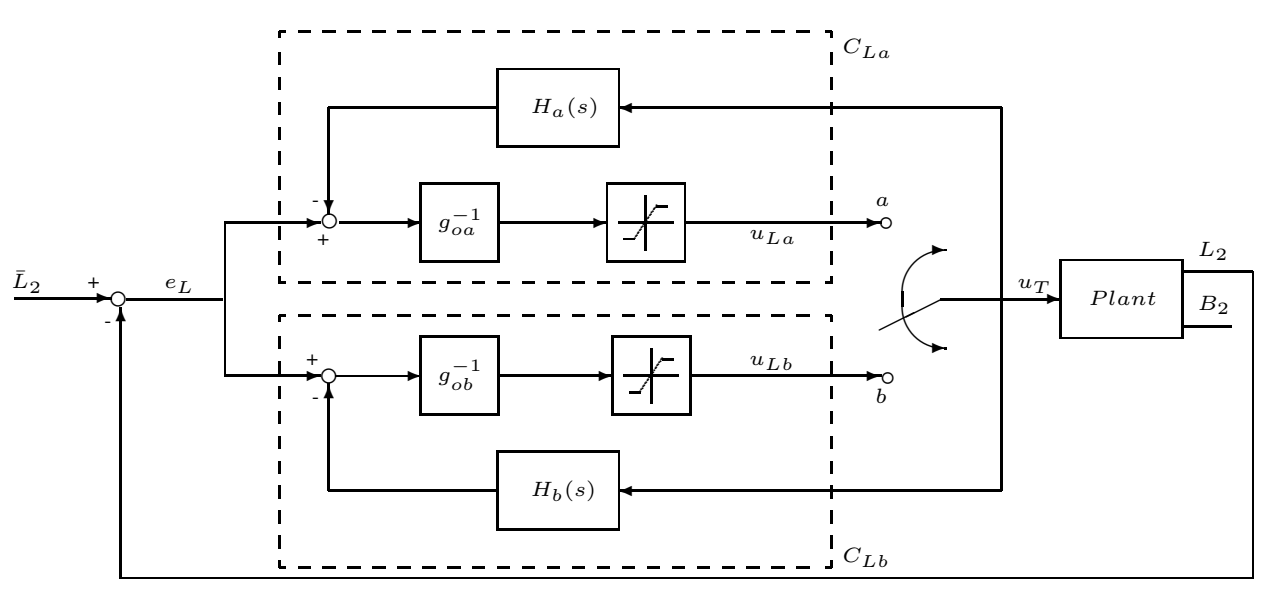

Fig. 4. Anti-wind-up and switching scheme implementation for control design testing.

controller $C_{B}(s)$ and the Level controller $C_{L}(s)$.

Notice from Figure 5 that when the switch is in position $a$ we have that $C_{L}(s)$ is the active controller and a copy of the brix controller $C_{B}(s)$ is in waiting as the latent controller. Viceversa, when the switch is in the $b$ position then $C_{B}(s)$ becomes the active controller and $C_{L}(s)$ the latent controller.

Remark 1: The achievement of BT in the SW-AWBT scheme relays on $e_{L} \approx 0$ and $e_{B} \approx 0$, since we can observe from Figure 5 that the latent controller, in comparison to the active controller, is always partially driven by a different error signal.

The above remark is not a great limitation for our proposed SW-AWBT scheme since the control authority swapping is meant to take place during nominal operation, thus implicitly granting small errors in both level and brix. This fact can be clearly observed in the second simulation presented in the next section.

Remark 2: We have assumed for both Figure 4 and Figure 5 that $u_{T}$ is directly a flow (in particular $F_{i n}$ ). This is a simplification without loss of generality, since the valve dynamics (see Figure 1) can always be considered as part of the plant model.

\section{Simulation Results}

In this section we explore the use of our proposed simple AWBT scheme through simulations of the MEE stage model (9) introduced in the previous section.

Initially we consider switching between different controllers having the same inputs and outputs. We then consider the case when we change the control configuration as described in Section II.

Simulation 1 (Switching controllers). The initial controllers are

$$
C_{L a}(s)=5.4\left(1+\frac{1}{600 s}\right), C_{B a}(s)=3.3\left(1+\frac{1}{660 s}\right),
$$

We will then transfer to the following controllers,

$$
C_{L b}(s)=5.4\left(1+\frac{1}{600 s}\right), C_{B b}(s)=0.5\left(1+\frac{1}{660 s}\right)
$$

as latent controllers, to be switched at time $t=50(\mathrm{~min})$ and back around at $t=216$ ( $\mathrm{min}$ ).

The level controller is the same in both pairs, and that the choice of brix controller is such that we should observe a difference in the closed loop behaviour.

In Figure 6 we show the system response with no BT mechanism in place for the brix controller only.

Observe from Figure 6, that the brix response of the system is affected by a large transient due to the controllers switching from pair $a$ to pair $b$, at $t=50(\mathrm{~min})$, and from pair $b$ back to pair $a$ at $t=216(\mathrm{~min})$. Indeed, notice from the second panel in Figure 6, how the latent control output does not track the active control output if no BT scheme is in place. For completeness we show in the third panel of Figure 6 the controller selection signal.

In Figure 7, we show the system response with the proposed BT mechanism in place. Again only the brix controller response is shown.

Observe from Figure 7, that the brix response of the system is unaffected by the controller switch at $t=50(\mathrm{~min})$, and at $t=216(\mathrm{~min})$. From the second panel in Figure 7 we can see the effect of the simple AWBT scheme proposal: the latent control output tracks the active control output reasonably well.

From Figure 6 and Figure 7, we see that our proposed AWBT scheme achieves bumpless transfer.

Simulation 2 (Exchanging controllers). Consider now the effect of exchanging the brix and level controls such that, as mentioned in the previous section, the level is controlled via the outflow valve, and the brix is controlled via the inflow valve. For this scenario we only make use of the pair of controllers in (10). For the inlet flow we start with $C_{L a}(s)$ driven by the level error as the active controller and a copy of $C_{B a}(\mathrm{~s})$ acts as the latent inlet controller. At $t=50(\mathrm{~min})$ $C_{B a}(s)$ is made active driven by the brix error and $C_{L a}(s)$ becomes the latent controller. Finally, at $t=216(\mathrm{~min})$, the controllers are switched to the initial configuration.

In Figure 8 the overall process response without the SWAWBT scheme is shown. The upper panel shows brix and level outputs, the middle panel shows the level controller 


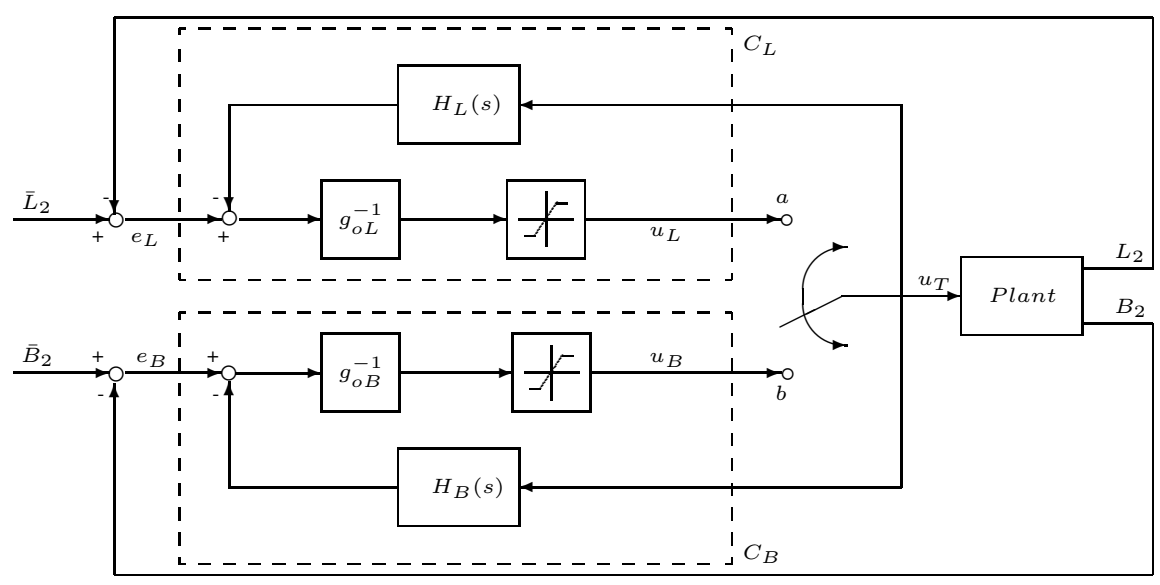

Fig. 5. Anti-wind-up and switching scheme implementation for control authority swapping.
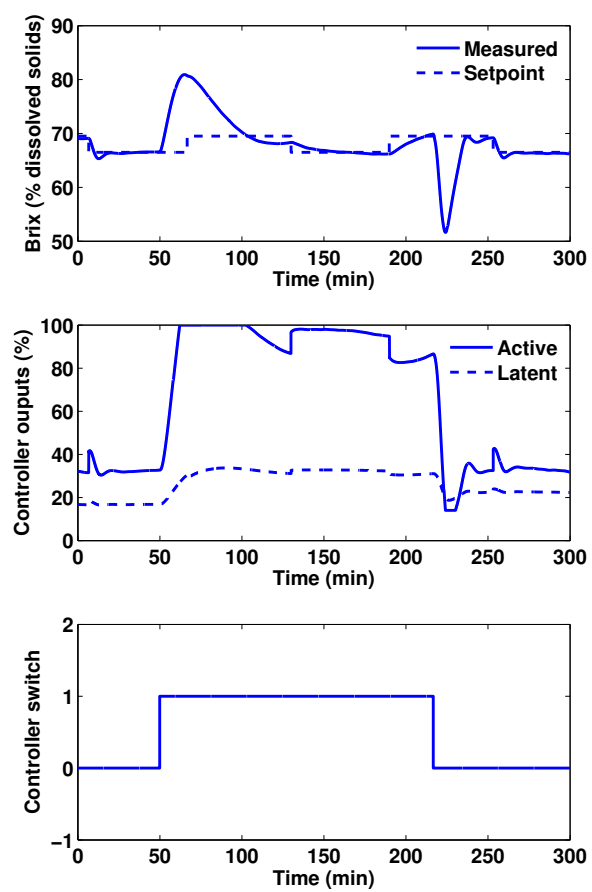

Fig. 6. Switching controllers MEE last stage response without the AWBT scheme (switching at $t=50(\mathrm{~min})$ and $t=216(\mathrm{~min})$ ).

outputs, and the lower panel the brix controller outputs. We observe from Figure 8 that, as expected when exchanging controllers, a bump is visible at $t=50(\mathrm{~min})$ and at $t=$ 216 ( $\mathrm{min}$ ), It is obvious that the latent controller outputs are not tracking their respective active controller outputs.

We then incorporate the proposed SW-AWBT scheme to the reversing scenario. Figure 9 shows the overall process response with the SW-AWBT scheme in place. The upper panel displays the brix and level outputs, the middle panel shows the level controller outputs, and the lower panel the brix controller outputs. We can observe from Figure 9 that


Fig. 7. Switching controllers MEE last stage response with the AWBT scheme (switching at $t=50(\mathrm{~min})$ and $t=216(\mathrm{~min})$ ).

the previously observed bumps at $t=50(\mathrm{~min})$ and at $t=216$ ( $\min$ ) have disappeared, thus achieving bumpless transfer. It can also be seen that the latent controller outputs track their respective active controller outputs. Figure 9 suggests then that the proposed SW-AWBT scheme might prove useful in achieving effective BT, not only for its original purpose of switching between different controller designs, but also for the more complex case of exchanging control authority. 



Fig. 8. Swapping controllers MEE last stage response without the SWAWBT scheme (switching at $t=50(\mathrm{~min})$ and $t=216(\mathrm{~min})$ ).


Fig. 9. Swapping controllers MEE last stage response with the SW-AWBT scheme (switching at $t=50(\mathrm{~min})$ and $t=216(\mathrm{~min})$ ).

\section{Final REMARKS}

In this paper we have proposed a simple bumpless transfer scheme and have shown its applicability through simulations with a highly detailed model of a sugar mill multi-effect evaporator stage. The simulation results provide solid evidence to propose the modification of the existing control architecture for the Multi-Effect Evaporators stage at Pioneer Sugar Mill, QLD, Australia. It is intended to apply these modifications during the 2009 cane crushing season.

The proposed method's application will allow on-the-fly testing of different controller designs and swapping of the control authority, whilst at the same time maintaining the existing anti-wind-up mechanisms. We are confident that the future application of such control architecture changes will result in improved performance of the real plant.

\section{REFERENCES}

[1] G.J. Adams, B.J. Burke, G.C. Goodwin, J.T. Gravdahl, R.D. Peirce, and A.J. Rojas. Managing Steam and Concentration Disturbances in Multi-Effect Evaporators via Nonlinear Modelling and Control. In Proceedings of the 17th IFAC World Congress, pages 13919-13924, Seoul, South Korea, July 2008.

[2] J.D. Bendtsen, J. Stoustrup, and K. Trangbaek. Bumpless Transfer Between Advanced Controllers with Applications to Power Plant Control. In Proceedings of the 42th IEEE Conference on Decision and Control, pages 2059-2064, Maui, Hawaii, USA, December 2003.

[3] C. Edwards and I. Postlethwaite. Anti-Windup and Bumpless Transfer Schemes. Automatica, 34(2):199-210, February 1998.

[4] S.S. Ge and Z. Sun. Switched Controllability via Bumpless Transfer Input and Dwell-time Switching. In Proceedings of the 46th IEEE Conference on Decision and Control, pages 714-719, New Orlean, LA, USA, December 2007.

[5] G.C. Goodwin, S.F. Graebe, and M.E. Salgado. Control System Design. Prentice Hall, 2001.

[6] S.F. Graebe and A.L.B. Ahlen. Dynamic Transfer Among Alternative Controllers and Its Relation to Antiwindup Controller Design. IEEE Transactions on Control Systems Technology, 4(1):92-99, January 1996.

[7] R. Hanus, M. Kinnaert, and J.-L. Henrotte. Conditioning Technique, a General Anti-windup and Bumpless Transfer Method. Automatica, 23(6):729-739, November 1987.

[8] I. Kaminer, A.M. Pascoal, P.P. Khargonekar, and E.E. Coleman. A Velocity Algorithm for the Implementation of Gain-scheduled Controllers. Automatica, 31(8):1185-1191, August 1995.

[9] E.F. Mulder, M.V. Kothare, and M. Morari. Multivariable anti-windup controller synthesis using linear matrix inequalities. Automatica, 37(9):1407-1416, September 2001.

[10] Y. Peng, D. Vrancic, and R. Hanus. Anti-Windup, Bumpless, and Conditioned Transfer Techniques for PID Controllers. IEEE Control Systems Magazine, 16(4):48-57, August 1996.

[11] Y. Peng, D. Vrancic, R. Hanus, and S.R. Weller. Anti-Windup Designs for Multivariable Controllers. Automatica, 34(12):1559-1565, December 1998.

[12] M.C. Turner and D.J. Walker. Linear quadratic bumpless transfer. Automatica, 36(8):1089-1101, August 2000.

[13] L. Zaccarian and A.R. Teel. A common framework for anti-windup, bumpless transfer and relaible designs. Automatica, 38(10):17351744, October 2002.

[14] L. Zaccarian and A.R. Teel. The $\mathcal{L}_{2}\left(l_{2}\right)$ bumpless transfer problem for linear plants: its definition and solution. Automatica, 41(7):12731280, July 2005.

[15] K. Zheng, T. Basar, and J. Bentsman. $H_{\infty}$ Bumpless Transfer under Controller Uncertainty. In Proceedings of the 46th IEEE Conference on Decision and Control, pages 2129-2134, New Orlean, LA, USA, December 2007. 